SHORT REPORT

\title{
Generalised anhidrosis: different lesion sites demonstrated by microneurography and skin biopsy
}

\author{
V Donadio, P Montagna, M Nolano, P Cortelli, C Misciali, G Pierangeli, V Provitera, A Casano, \\ A Baruzzi, R Liguori
}

J Neurol Neurosurg Psychiatry 2005;76:588-591. doi: 10.1136/jnnp.2004.039263

Generalised anhidrosis (GA) shows a uniform clinical picture whether the pathogenesis involves intrinsic abnormalities of sweat glands or postganglionic sympathetic cholinergic nerve dysfunction. We describe two patients who presented intolerance to heat and anhidrosis. In the first patient, symptoms started at 33 years of age, and were associated with absent tendon reflexes and a mydriatic right pupil unreactive to light. The other patient had been unable to sweat since birth. GA was diagnosed on the basis of clinical findings and thermoregulatory tests. Microneurography and morphological analysis of the skin and its innervation disclosed a different lesion site underlying GA in the two patients, and distinguished between a postganglionic autonomic nerve fibre lesion and sweat gland dysfunction.

G eneralised anhidrosis (GA) is a rare congenital or acquired disease characterised by heat intolerance and loss of sweating, and may be associated or not with autonomic peripheral neuropathies..$^{1-5}$ The clinical picture in GA is uniform, whether pathogenesis involves intrinsic sweat gland abnormalities ${ }^{2}{ }^{6}$ or postganglionic sympathetic cholinergic nerve dysfunction. ${ }^{35}$ Laboratory studies are therefore needed to localise the specific lesion site. The sudomotor axon reflex test can distinguish between preganglionic and postganglionic sudomotor lesions but it cannot differentiate postganglionic sudomotor outflow from sweat gland abnormalities.'

We studied by means of microneurography and skin biopsy two GA patients in whom a different pathogenesis was suspected, in order to establish the lesion site responsible for their anhidrosis.

\section{PATIENTS}

\section{Patient 1}

A 38 year old woman had noticed, at the age of 31 years, that her right pupil was larger than the left. At 32 years of age, during the summer, she developed general fatigue and intolerance to heat and, during exercise, excessive sweating over the right shoulder and neck, the other body surfaces remaining dry. In 1 year, she lost the ability to sweat all over her body and was confined to an indoor environment during summer. Neurological examination showed absent tendon reflexes and a mydriatic right pupil, unreactive to light. Diluted pilocarpine $(0.062 \%)$ and adrenalin $(0.1 \%)$ induced in the right pupil a constriction of $1.92 \mathrm{~mm}$ after 45 minutes and a dilatation of $1.20 \mathrm{~mm}$ after 30 minutes, but had no effect on the left pupil, indicating a postganglionic parasympathetic and sympathetic denervation of the right pupil. Schirmer test was normal. Two months of methylprednisolone therapy (75 mg/day) did not improve sweating.

\section{Patient 2}

A 46 year old woman, unable to sweat since birth, complained of hyperthermia with skin burning and itching, tachycardia, photophobia, and phonophobia, followed by headache when ambient temperature was above $34^{\circ} \mathrm{C}$. Family history and neurological examination were unremarkable. We previously reported sweat gland abnormalities in this patient but postganglionic sympathetic innervation to sweat glands was not studied. ${ }^{7}$

\section{Testing}

In both patients, screening for autoimmune, microbiological, and neuroendocrinological diseases was negative. EEG, brain MRI, nerve conduction studies, and cardiovascular reflexes (tilt test, Valsalva manoeuvre, deep breathing, and handgrip tests) were normal.

\section{METHODS}

The study was fully explained to the patients, who gave their written consent to the procedures proposed.

\section{Thermoregulatory tests}

Thermoregulatory sweat test (TST) and circadian rhythm of body core temperature (CRT) were performed as previously described. CRT was evaluated by monitoring rectal temperature continuously every 2 minutes for 48 hours in a room with controlled mean (SD) temperature of $24(1)^{\circ} \mathrm{C}$ and humidity of $40-50 \% .^{78}$

\section{Microneurography recordings}

Patients sat in an ambient temperature of $25^{\circ} \mathrm{C}$ and humidity of $30 \%$ in a semi-dark soundproofed room. Multiunit recordings of efferent postganglionic sympathetic nerve activity were obtained from the left peroneal nerve, posterior to the fibular head. ${ }^{9}$ Muscle sympathetic nerve activity (MSNA) was considered acceptable when it revealed spontaneous, pulse synchronous bursts of neural activity that fulfilled the criteria previously described..$^{10}$ A burst of skin sympathetic nerve activity (SSNA) was considered to occur if it: (a) showed irregular occurrence varying in strength and duration, unrelated to heartbeats; $(b)$ at rest was followed by changes in finger pulse amplitude and/or skin electrical potential; and $(c)$ was evoked by various arousal stimuli, including surface electrical stimulation. To be included as an integrated SSNA burst, the amplitude had to exceed 5\% of the highest burst.

Abbreviations: AIGA, acquired idiopathic generalised anhidrosis; CED, congenital ectodermal dysplasia; CRT, circadian rhythm of body core temperature; ENF, epidermal nerve fibre; GA, generalised anhidrosis; IPSF, idiopathic pure sudomotor failure; MSNA, muscle sympathetic nerve activity; RS, Ross syndrome; SGF, sweat gland failure; SSNA, skin sympathetic nerve activity; TST, thermoregulatory test; VIP, vasoactive intestinal peptide 


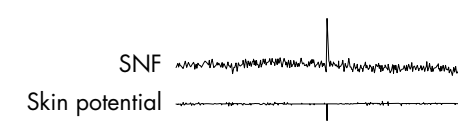

Skin blood flow Munumunumumumumumumumun

Resp.

Integrated MSNA unMUWMUWWWhWN

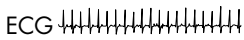

B

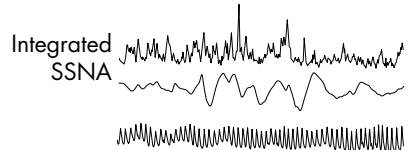

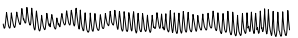

m

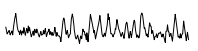

the
C
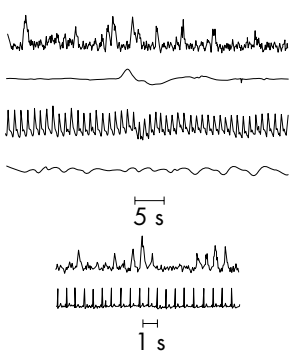

Figure 1 Microneurography recordings of skin (upper panel) and muscle (lower panel) nerve fascicles from left peroneal nerve in (A, B) patients 1 and 2 with GA and in (C) a healthy control subject. Patient 1: SSNA burst, skin potential and skin flood flow changes were not detected even after maximum surface electrical stimulation to the wrist $(99 \mathrm{~mA}$ intensity and $1 \mathrm{~s}$ duration as indicated by arrow), whereas MSNA bursts showing normal cardiac rhythmicity were recorded. Patient 2: spontaneous SSNA bursts were recorded, which showed a fairly high resting rate with a prevalence of sudomotor activity. Spontaneous, pulse synchronous MSNA bursts were recorded. Control: spontaneous SSNA bursts were recorded showing a lower resting rate compared with patient 2 and with a prevalence of vasomotor activity. MSNA showed normal characteristics. SNF, skin nerve fascicle; Resp, thoracic respiration.

Investigation for SSNA and MSNA bursts was performed on the same day by changing nerve fascicles. The procedure lasted a maximum of 60 minutes. Skin blood flow by infrared photoelectric transducer (model PPS; Grass Instruments) and changes in skin potential by Ag-AgCl surface electrodes (filter setting $0.2-100 \mathrm{~Hz}$ for both) were measured in the corresponding impaled skin fascicles. After acquiring a stable recording site, resting SSNA and MSNA were recorded for 20 minutes, and the last 5 minutes were analysed. Sudomotor and vasomotor nerve activities of SSNA were separately measured at rest, evaluating a burst followed by a change in isolated skin potential (sudomotor activity) and a burst followed by a change in isolated skin blood flow (vasomotor activity). Resting SSNA was followed by a 3 minute mental stress task using arithmetic subtraction.

\section{Skin biopsy}

To visualise skin nerve fibres, $3 \mathrm{~mm}$ punch biopsies were taken from fingertip, leg, and thigh. As previously described, ${ }^{11}$ specimens were fixed, sectioned, and incubated with primary antibodies, mainly to the pan-neuronal marker protein gene product 9.5 (PGP 9.5, 1:800; Biogenesis, Poole, UK) and to the autonomic fibre marker vasoactive intestinal peptide (VIP, 1:1000; Incstar, Stillwater, MN, USA); Ulex europaeus agglutinin I (UEA I) was used to visualise the endothelium. Digital images were acquired using a confocal microscope and epidermal nerve fibre (ENF) density per linear millimetre of epidermis was calculated.

Light microscopic histopathological examination of the sweat glands was performed on skin samples taken from the right forearm.

\section{RESULTS}

\section{Thermoregulatory tests}

In both patients, TST disclosed complete anhidrosis, except for small patchy areas of sweating in the axillae and neck of patient 1. CRT showed a stable circadian rhythm with normal mesor (patients $\mathrm{l}$ and 2 respectively: $37^{\circ} \mathrm{C}$ and $36.92^{\circ} \mathrm{C}$; normal $\left.37(0.2)^{\circ} \mathrm{C}\right)$, rhythm amplitude $\left(0.22\right.$ and $0.49^{\circ} \mathrm{C}$; normal $0.38(0.15)^{\circ} \mathrm{C}$ ) and acrophase (16 hours and
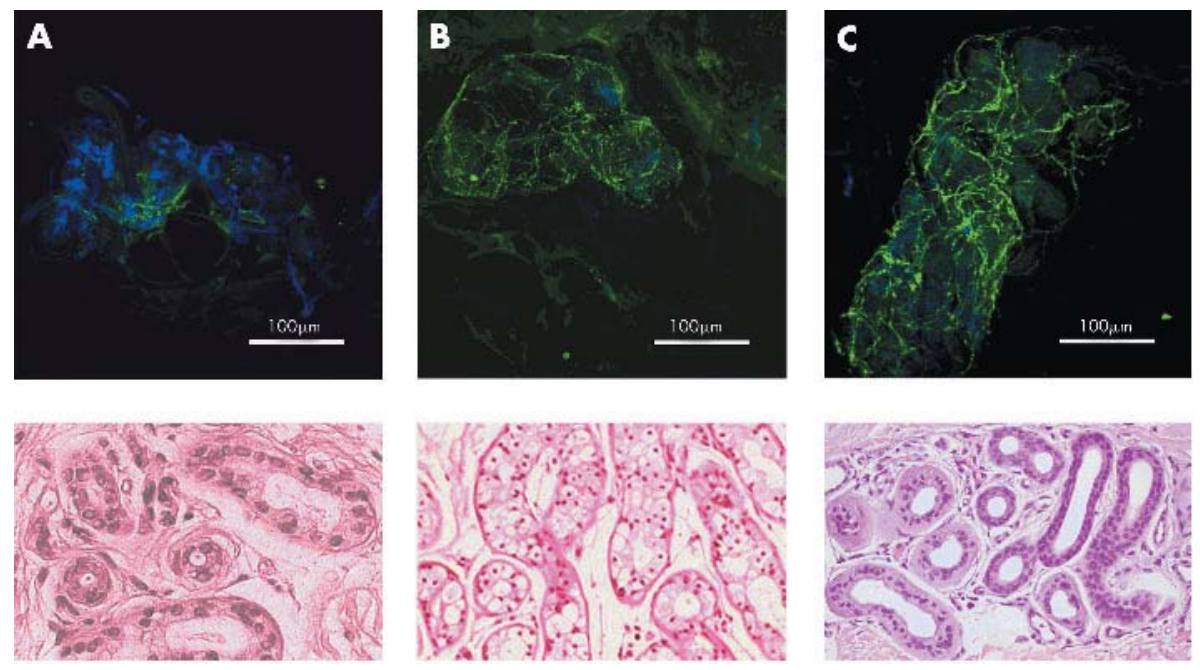

Figure 2 Double stained confocal images (upper panel) of sweat gland innervation from leg (VIP immunoreactive fibres in green; Ulex europaeus used to visualise the endothelium in blue) and light microscopy images (lower panel) of sweat gland from forearm (haematoxylin and eosin, original magnification $\times$ 40) in (A, B) patients 1 and 2 with GA and (C) the control subject. Patient 1: cholinergic VIP immunoreactive fibres were nearly absent (in green; upper panel) around the sweat gland; histology of the gland was normal (lower panel). Patient 2: a number of VIP immunoreactive fibres were detected around the sweat gland (in green; upper panel), which showed histopathological aspects of incomplete development (general disorganisation and substitution of sweat cells) compatible with dysplasia (lower panel). Control: rich VIP immunoreactivity was detected around the sweat gland (in green; upper panel), which showed normal histological findings (lower panel). 
15.87 hours; normal 15.80 (1.13) hours) (normal values are mean $(\mathrm{SD}))$.

\section{Microneurography recordings}

In patient 1, several skin fascicles were impaled but, after searching for approximately $45 \mathrm{~min}$, we were unable to record SSNA burst at rest, during mental stress, or after applying a maximum surface electrical stimulation (99 mA intensity and 1 second duration). MSNA was recorded and the mean level was 35 bursts/min and 53 bursts/100 heartbeats (normal 38 (3) and 54 (4)) (fig 1).

In patient 2, SSNA bursts were recorded with a mean resting rate of 12 bursts/min (normal 9 (1) bursts/min), mainly of sudomotor outflow (46\% sudomotor and $11 \%$ vasomotor activity of the total bursts; normal 5 (1) and 43 (3)\%), slightly increased by mental stress ( 15 bursts/min; normal 14 (3) bursts/min). MSNA showed a normal mean resting level (39 bursts/min and 56 bursts/100 heartbeats) (fig l).

\section{Skin biopsy}

In normal subjects, PGP 9.5 reveals an even distribution of nerve fibres in the epidermis, subepidermal neural plexus, and around dermal annexes. ${ }^{12}$ Sweat glands appear densely innervated by VIP immunoreactive fibres (fig 2). ${ }^{13}$

In patient 1 , VIP immunoreactive fibres were virtually absent around sweat glands, which appeared substantially normal without microscopic abnormalities or infiltrates on histopathological analysis (fig 2). ENF density was slightly reduced in the fingertip (4; normal 13.1 (4)) and thigh (16; normal $27(6))$, and at the lower limit of normal range in the leg (12; normal $17.4(3))$. A mild loss of nerve fibres was observed in the subepidermal plexus and around blood vessels.

In patient 2, sweat glands appeared markedly atrophic on histopathology and presented microscopic changes compatible with dysplasia without inflammatory infiltrates. A number of VIP immunoreactive fibres were detected around sweat glands (fig 2), and ENF density was normal in fingertip (11.1), thigh (25), and leg (20).

\section{DISCUSSION}

The lesion sites causing GA are heterogeneous and estimated to be at several levels: (a) hypothalamic thermoregulatory area; ${ }^{14}(b)$ autonomic neuropathy involving sudomotor nerves; ${ }^{3}$ (c) idiopathic pure sudomotor failure (IPSF) with neurotransmission failure from terminals to effector organs, ${ }^{15}$ $(d)$ sweat gland failure (SGF); ${ }^{16}$ and $(e)$ congenital ectodermal dysplasia (CED).

Acquired idiopathic generalised anhidrosis (AIGA) involves mainly young people with no other associated major autonomic disorders. The elevated levels of serum IgE and lymphocyte infiltration around sweat glands suggested an immunological pathogenesis. ${ }^{16}$ IPSF and SGF are included in the AIGA syndrome and are predominant in Japanese and other East Asian people. In addition to anhidrosis, ISPF presents characteristic onset symptoms such as skin dysaesthesia, itching, and red eruptions, and the pathogenesis involves a steroid responsive immunological process that inhibits the transduction signal of cholinergic nerves to sweat gland receptors, altering the muscarinic receptor of acetylcholine. ${ }^{15}$ SGF is characterised by sweat gland immunological deposits and by a poor response to steroids. ${ }^{16}$

Immunologically related anhidrosis characterising AIGA is different from anhidrosis caused by CED or Ross syndrome (RS), which involves a degenerative pathogenesis without immunological implication. CED shows anomalies of sweat glands, which may be associated with hair, skin, or dental abnormalities, ${ }^{2}$ whereas RS is characterised by a selective degeneration of autonomic and dorsal root ganglia neurones but its cause remains unknown. ${ }^{17}$

Our patients had GA as indicated by the clinical findings and TST. Both patients also had normal brain MRI and displayed a normal CRT, which excluded a hypothalamic lesion site. Microneurography and skin biopsy disclosed the lesion site, which differed in the two patients.

In patient 1, microneurography failed to record an SSNA burst, and skin innervation analysis disclosed absent sudomotor VIP immunoreactive fibres around sweat glands and a moderate reduction of PGP 9.5 immunoreactive fibres to blood vessels. Together with the normal findings upon histopathological examination of the sweat glands, this suggested a postganglionic sympathetic lesion (autonomic neuropathy) as the cause of anhidrosis. The clinical picture (tonic pupil, areflexia, and anhidrosis) and the underlying lesion of autonomic postganglionic fibres suggested a diagnosis of RS in this patient. ${ }^{17}$

Patient 2 showed SSNA bursts increasing during mental stress and a preserved autonomic innervation of sweat glands and blood vessels. Microscopic examination, however, detected markedly atrophic sweat glands, which showed morphological changes compatible with dysplasia as the underlying cause of anhidrosis. The patient's resting SSNA was increased, with a higher prevalence of sudomotor activity compared with controls, possibly due to a thermoregulatory feedback mechanism to compensate for the absence of sweat expulsion. ${ }^{16}$ Clinical and laboratory findings thus indicated that patient 2 had CED selectively involving the eccrine glands. ${ }^{2}$

The normal cardiovascular reflexes and MSNA findings in both patients excluded a more diffuse involvement of the autonomic nervous system, rarely described in GA. ${ }^{18}$

In conclusion, microneurography associated with morphological analysis of sweat glands and their innervation proved reliable diagnostic tools in detecting the site of the autonomic dysfunction responsible for GA in our patients, and in differentiating between a postganglionic autonomic nerve fibre lesion and sweat gland dysfunction.

\section{Authors' affiliations}

V Donadio, P Montagna, G Pierangeli, A Casano, A Baruzzi, R Liguori, Department of Neurological Sciences, University of Bologna, Via U. Foscolo 7, 40123 Bologna, Italy

M Nolano, V Provitera, Department of Neurology, Salvatore Maugeri Foundation, IRCCS, Via Bagni Vecchi, 82037 Telese Terme (BN), Italy P Cortelli, Department of Neurology, University of Modena and Reggio Emilia, Via Dal Pozzo, 41100 Modena, Italy

C Misciali, Department of Dermatology, S. Orsola Hospital, Via Massarenti 9, 40135 Bologna, Italy

Competing interests: none declared

Correspondence to: Dr V Donadio, Dipartimento di Scienze Neurologiche dell'Università di Bologna, Via Ugo Foscolo 7, 40123 Bologna, Italy; donadio@neuro.unibo.it

Received 13 February 2004

In revised form 12 July 2004

Accepted 4 August 2004

\section{REFERENCES}

1 Low PA, Fealey RD, Sheps SG, et al. Chronic idiopathic anhidrosis. Ann Neurol 1985; 18:344-8.

2 Kleinebrecht J, Degenhardt N, Grubysic A, et al. Sweat pore counts in ectodermal dysplasia. Hum Genet 1981;57:437-9.

3 Miyazoe S, Matsuo H, Ohnishi A, et al. Acquired idiopathic generalized anhidrosis with isolated sudomotor neuropathy. Ann Neurol 1998;44:378-81.

4 Ross AT. Progressive selective sudomotor denervation: a case with coexisting Adie's syndrome. Neurology 1958;8:809-17. 
5 Nolano M, Crisci C, Santoro L, et al. Absent innervation of skin and sweat glands in congenital insensitivity to pain with anhidrosis. Clin Neurophysiol 2000;111:1596-601

6 Ando Y, Fujii S, Sakashita N, et al. Idiopathic acquired generalized anhidrosis: clinical manifestations and histochemical studies. J Neurol Sci 1995; 132:80-3.

7 Cevoli S, Pierangeli G, Magnifico F, et al. The circadian rhythm of body core temperature (CRT) is normal in patients with congenital generalised anhidrosis. Clin Auton Res 2002;12:170-3.

8 Magnifico F, Pierangeli G, Barletta G, et al. Paroxysmal episodic central thermoregulatory failure. Neurology 2002;58:1300-2.

9 Wallin BG. Assessment of sympathetic mechanisms from recordings of postganglionic efferent nerve traffic. In: Hainsworth R, Mark AL, eds. Cardiovascular reflex control in health and disease. WB Saunders: London, 1994:65-93.

10 Sundlof G, Wallin BG. The variability of muscle nerve sympathetic activity in resting recumbent man. J Physiol 1977;272:383-97.
11 Kennedy WR, Wendelschafer-Crabb G. The innervation of human epidermis. J Neurol Sci 1993; 115:184-190.

12 Nolano M, Provitera V, Crisci C, et al. Small fibers involvement in Friedreich's ataxia. Ann Neurol 2001:50:17-25.

13 Perretti A, Nolano M, De Joanna G, et al. Is Ross syndrome a dysautonomic disorder only? An electrophysiologic and histologic study. Clin Neurophysiol 2003;114:7-16.

14 Shimizu H, Obi T, Miyajima H. Anhidrosis: an unusual presentation of diabetes insipidus. Neurology 1997;49:1708-10.

15 Nakazato $Y$, Shimazu K, Tamura N, et al. Idiopathic pure sudomotor failure. Rinsho Shinkeigaku 1994;34:12-15.

16 Muramaki K, Sobue G, Iwase S, et al. Skin sympathetic nerve activity in acquired idiopathic generalized anhidrosis. Neurology 1993;43:1137-40.

17 Sommer C, Lindenlaub T, Zillikens D, et al. Selective loss of cholinergic sudomotor fibers causes anhidrosis in Ross syndrome. Ann Neurol 2002;52:247-50.

18 Bacon PJ, Smith SE. Cardiovascular and sweating dysfunction in patients with Holmes-Ádie syndrome. J Neurol Neurosurg Psychiatry 1993;56:1096-102. 